\title{
CAS and PSI increase coronal alignment accuracy and reduce outliers when compared to traditional technique of medial open wedge high tibial osteotomy: a meta-analysis
}

\author{
S. Cerciello ${ }^{1,2} \cdot$ M. Ollivier ${ }^{3} \cdot$ K. Corona ${ }^{4}$ D $\cdot$ B. Kaocoglu ${ }^{5} \cdot$ R. Seil ${ }^{6,7,8}$
}

\begin{abstract}
Purpose Medial open-wedge high tibial osteotomy (MOWHTO) is an accepted option in the treatment of medial compart-ment osteoarthritis of the knee in young and active patients. Functional results are closely correlated to the correction of the mechanical axis of the lower limb. Although several angular and geometrical methods and values have been proposed in the past, the ideal target is still debated. In addition, it is important to have a deep correlation between the planned correction and the achieved correction after surgery. The aim of the present systematic review was to identify the ideal coronal correction after MOWHTO and the most accurate method to achieve it.

Methods A systematic review of the literature was completed on July 3rd 2020 in the Pubmed, Medline, Cochrane Reviews, and Google Scholar databases using the Medical Subject Headings (MeSH) terms: "high tibial osteotomy" AND "accuracy" OR "planning".

Results 28 studies were included; 18 were focused on computer-assisted surgery (CAS) and 10 on patient-specific instrumentation (PSI). There were 598 patients in the CAS group and 501 in the control group; the rate of outliers was $16 \%$ and $38.2 \%$ respectively $(P=0.04)$, while there was no significant difference between the two groups (SMD $=-0.10 ; 95 \%$ CI 1.31 to $1.12 ; P=$ n.s.) in terms of coronal accuracy. Likewise, there were 318 patients in the PSI group and 40 in the control group; the rate of outliers was $15 \%$ and $40 \%$ respectively $(P=0.98)$, while there was no significant difference between the two groups (SMD $=0.01 ; 95 \% \mathrm{CI} 0.58$ to $0.59 ; P=0.98$ ).

Conclusions A statistically significant reduced outlier rate and a non-significant increased accuracy emerged with the use of CAS when compared to the traditional surgical technique, whereas the results of PSI were still inconclusive. In addition, it emerged clearly that no consensus still exists on the ideal correction target to be achieved after surgery.

Level of evidence III.
\end{abstract}

Keywords High tibial osteotomy · Medial open wedge · Accuracy · Coronal alignment · Computer assisted surgery · Patient specific instrumentation 


\section{Introduction}

Medial open-wedge high tibial osteotomy (MOWHTO) is a well-recognized option in the treatment of medial compartment osteoarthritis of the knee, medial compartment overload or spontaneous osteonecrosis of the medial femoral condyle $[1,2]$. It was also been proposed in association with ligament reconstruction or meniscal transplantation in varus knees with encouraging outcomes [24]. The MOWHTO aims to correct an extra-articular deformity, shifting the weight bearing line from the medial compartment to a more lateral position, thus unloading cartilage and subchondral bone.

The clinical outcomes are often satisfactory, even at longterm follow-up, with 10 and 15-year survival rates of $92 \%$ and $71 \%$, respectively [37]. However, the results depend on the accurate correction of the lower limb alignment according to the preoperative plan [16]. Historically, it was suggested to achieve $8^{\circ}-10^{\circ}$ of postoperative valgus in the anatomical axis (AA) or $3^{\circ}-5^{\circ}$ of valgus in the mechanical axis (MA) [20]. Persistent varus axis can lead to recurrence of deformity whereas overcorrection can accelerate lateral compartment OA [20]. An angular correction of $3^{\circ}-5^{\circ}$ usually corresponds to a mechanical axis that crosses the tibial plateau at around $65-70 \%$ of its width (with $0 \%$ being the medial and $100 \%$ the lateral border). This area has been initially described by Fujisawa et al. [9]. More recently authors advocated for a postoperative mechanical axis (MA) of the lower limb of approximately of $2^{\circ}-7^{\circ}$ valgus which is the range usually associated with favorable clinical outcomes for cartilage repair $[17,18]$. Although the theoretical principles seem clear and easy, some fundamental aspects of high tibial medial wedge osteotomy, like the ideal postoperative target, the precision of planning and intraoperative accuracy have not yet clearly addressed.

The present systematic review and meta-analysis has therefore several aims. First of all, to compare the accuracy of new tencologies such as computer assisted surgery (CAS) and patient specific instrumentation (PSI) in achieving the postoperative correction target when compared to the tradional MOWHTO. Second, to compare the rate of postoperative outliers among the different surgical options. Finally to identify the ideal anatomic and angular target after MOWHTO. It was hypotesized that CAS and PSI were more accurate and yield less outliers than the traditional technique.

\section{Methods}

This systematic review was carried out in accordance with the recommendations proposed by the Preferred Reporting Items for Systematic Reviews and Meta-analyses (PRISMA) statement [29].

\section{Search strategy}

Multiple database, including MEDLINE, EMBASE and the Cochrane Library were searched on the 3rd of July 2020 for studies reporting the accuracy of different methods of planning (conventional, navigation and patient specific guides) in high tibial osteotomy. Medical Subject Headings (MeSH) terms used for the search included "medial open wedge high tibial osteotomy" AND/OR "accuracy" AND/OR "planning". After the initial electronic search which yielded 110 results, relevant articles and their bibliographies were searched manually. Articles identified were assessed individually for inclusion (Fig. 1).

\section{Study selection}

The following exclusion criteria were applied to the articles: (1) non-English literature; (2) biomechanical studies; (3) articles in which standard-procedure HTO, with the indication osteoarthritis and/or genu varum, was not the main objective; (4) case reports (less than 5 knees in the study), replies to other pubblications; (5) studies in which data on the accuracy of HTO, or the rate of outliers was unavailable. The remaining articles were included in this systematic review.

\section{Data extraction}

Each study was evaluated for the following variables: type of study, population size, gender and age, correction target in terms of Fujisawa area or angular correction, duration of the surgical procedure, follow-up (FU), accuracy of the achieved correction (in terms of Fujisawa area or angular correction), complications and outliers. All these information was searched and reported for three groups: patients treated with MOWHTO and traditional radiographic planning, patients treated with computer assisted MOWHTO and patients operated with the assistance of 3D CT analysis and patient specific instrumentation (PSI). All the articles were evaluated, and data extracted by two authors independently. Any differences in the sensible data between the two authors lead to a new analysis and a double check of the article. If any data was missing in one of the two authors evaluation, this lead to a new analysis and a double check of the article.

\section{Methodological quality assessment}

As no randomized controlled trials were found, the Methodological Index for Nonrandomized Studies (MINORS) checklist [39], a tool specifically created to evaluate the 


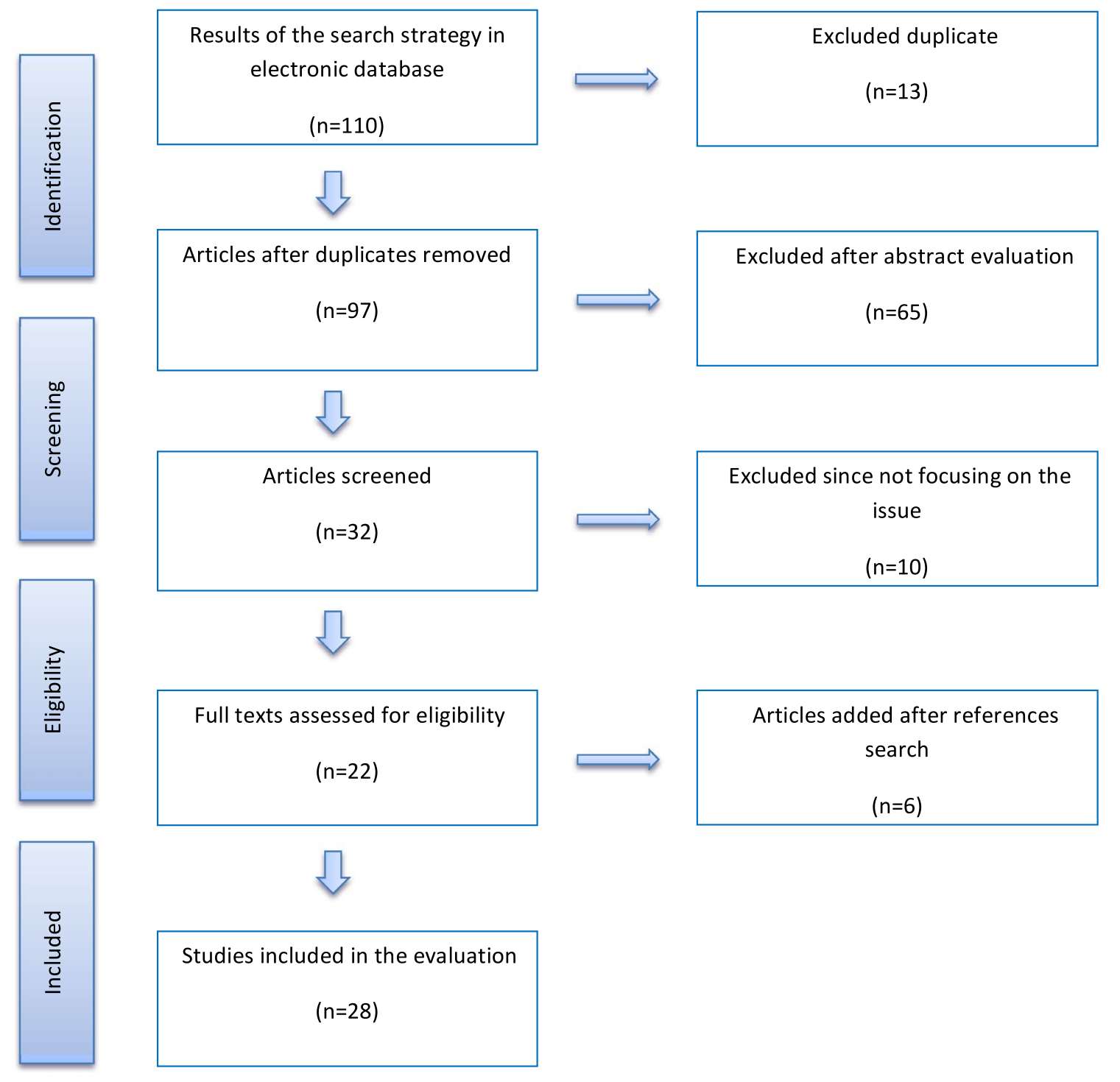

Fig. 1 PRISMA flow chart of the papers selection process

quality of nonrandomized surgical studies, was used to evaluate the quality of the included studies. The checklist includes 12 items, with the last 4 specific to comparative studies. Scoring was as follows: 0 , not reported; 1 , reported but poorly done and/or inadequate; and 2 , reported, well done and adequate. The highest overall score was 16 for noncomparative studies and 24 for comparative studies. Each study was scored by two authors (KC and SC) who reached consensus if disagreement occurred with the intervention of the third author (MO).

\section{Outcomes assessment}

The target of coronal alignment after MOWHTO was reported in terms of angular correction referred to the hipknee angle (HKA), femoro-tibial angle (FTA) or mechanical medial proximal tibial angle (mMPTA) or in terms of the rate of the lateral tibial plateau intersected by the lower limb mechanical axis. The difference between the preoperative target and the achieved correction (accuracy) was reported. In comparative studies the difference between navigated osteotomies and traditional technique and between 3D CT scan-based PSI and traditional technique was measured. The rate of outliers in terms of postoperative correction (if available), the duration of the surgical procedure (if available), the rate of complications (if available) were reported for the different groups.

\section{Statistical analysis}

Statistical analysis was performed with Review Manager (Version 5.3, The Cochrane Collaboration). Risk ratio (RR) 
and odds ratio (OR) were used as summary statistics to perform statistical analysis of dichotomous variables, and the mean difference (MD) was used to analyse continuos variables. They were reported with $95 \%$ confidence intervals (95\% CI), and $P$ value of 0.05 was used as the level of statistical significance. Statistical heterogeneity between trials was evaluated by the Chi square and $I$-square $\left(I^{2}\right)$ test, with significance set at $P<0.10$. An $\mathrm{I}^{2}$ above $40 \%$ was considered to be significant. The random effect model was used in the presence of significant heterogeneity, while in the absence of significant heterogeneity, the fixed effect was preferred. A sensitivity analysis was conducted by excluding one study in each round and evaluating the influence of any single study on the primary meta-analysis estimate.

\section{Results}

\section{Search results}

The electronic search yielded 110 studies. After 13 duplications were removed, 97 studies remained; of these, 65 were excluded after review of the abstracts and full-text articles leaving 32 eligible studies. An additional 10 articles were then excluded mainly based on the different ways to analyze the ouctcomes (uniquely on standard X-rays, or using the cable method alone or with a theoretical computer-method, or with picture archiving method). When manually checking the reference list of the included articles, 6 additional studies were found. Finally, 28 articles met the inclusion criteria: 18 were focusing on CAS compared to traditional technique and 10 on PSI compared to traditional technique. Figure 2 shows the flow chart of the study selection process.

\section{Methodological quality assessment}

The studies in the CAS/traditional technique subgroup had a mean MINORS score of 15.9 (range 10-18) while in the PSI/traditional subgroup the mean MINORS score was 12.6 (range 10-18), which confirmed the good methodological quality of the available literature (Tables 1,2).

\section{Patients and study characteristics}

Tables 3 and 4 show the characteristics of the studies and the demographic characteristics of the cohorts. When focusing on CAS/traditional technique 18 studies were available. There were 598 patients in the study (CAS) group and 501 in the control group. The groups were comparable in terms of age and male/female ratio. The targeted correction was extremely variable in the different studies (Table 1). When focusing on PSI/traditional technique 10 studies were available. There were 318 patients in the study (PSI) group and 40 in the control group. The groups were comparable in terms of age, the rate of female patients was higher in the control group (75\%). The targeted correction was extremely variable in the different studies (Table 2).

\section{Outcomes assessment: coronal correction and outliers}

In the CAS/traditional technique the pooled results of aggregate analysis for comparison of navigated and conventional HTO regarding accuracy of alignment, including subgroup analysis by the method of post-operative alignment: angular correction (mean \pm SD) and area (\%) described by Fujisawa,

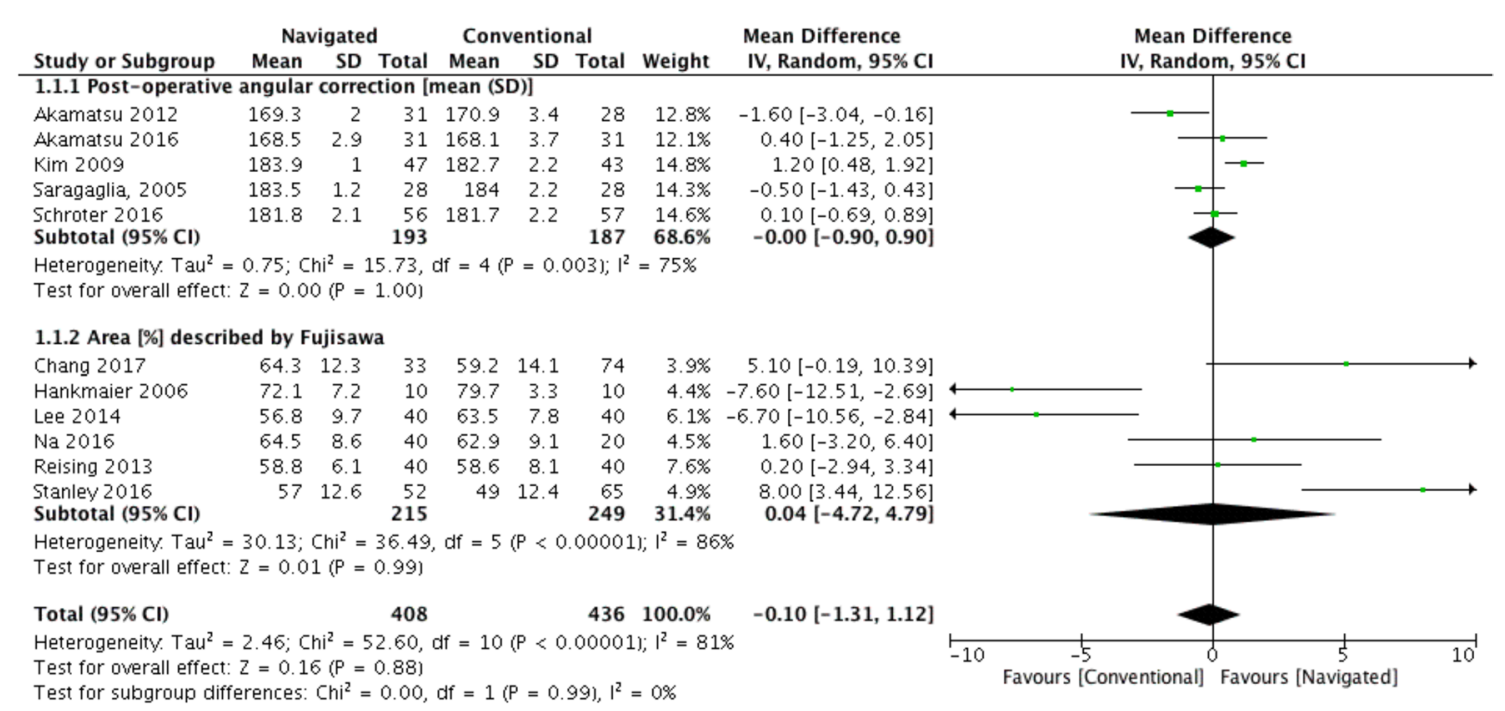

Fig. 2 Comparison of coronal correction in the computer assisted surgery (CAS)/traditional technique studies 
Table 1 Coronal correction of the computer-assisted surgery (CAS)/traditional technique studies

\begin{tabular}{|c|c|c|c|c|c|c|}
\hline Authors & SG & 1st target & 2nd target & FU months & Conclusions & Minors \\
\hline Saragaglia 2005 [36] & 28 & $182-186$ & & 3 & Better navigation & 14 \\
\hline Maurer 2006 [27] & 44 & $182-185$ & & & Better navigation & 16 \\
\hline $\begin{array}{l}\text { Hankemeier } 2006 \\
\text { [12] }\end{array}$ & 10 & $80 \%$ & & 0 & Better navigation & 18 \\
\hline Kim 2009 [19] & 47 & $183-185$ & 62 & 12 & Better navigation & 16 \\
\hline Lutzner 2010 [25] & 10 & $80 \%$ & & 0 & Better navigation & 18 \\
\hline Iorio 2011 [14] & 14 & $182-186$ & & 12.6 & No control & 12 \\
\hline Gebhard 2011 [10] & 51 & $182-183$ & & 1.5 & No control & 10 \\
\hline Lee 2012 [23] & 39 & $182-188$ & $62 \%$ & 6.5 & No control & 12 \\
\hline Akamatsu 2012 [2] & 31 & 190 & $167-173$ & 12 & Better navigation & 16 \\
\hline Reising 2013 [34] & 40 & $62 \%$ & & & No difference less outliers & 16 \\
\hline Iorio 2013 [13] & 14 & $182-186$ & & 39 & Better navigation & 18 \\
\hline Lee 2014 [22] & 40 & $62 \%$ & & 2 & Worse navigation & 18 \\
\hline Ribeiro 2014 [35] & 18 & $62 \%$ & $62 \%$ & 12 & No difference & 16 \\
\hline Akamatsu 2016 [1] & 31 & (FTA) 165-175 & $62 \%$ & 24 & Better navigation & 18 \\
\hline $\mathrm{Na} 2016$ [31] & 40 & $62 \%$ & & 3 & Better navigation & 16 \\
\hline Schröter 2016 [38] & 56 & $182-183$ & & 1.5 & No difference & 18 \\
\hline Stanley 2016 [40] & 52 & $58 \%$ & & & No difference & 16 \\
\hline Chang 2017 [3] & 33 & $62.5 \%$ & & 12 & Better navigation & 18 \\
\hline
\end{tabular}

$S G$ Study group, FTA femoro-tibial angle, $F U$ follow-up

\begin{tabular}{lllllll}
\hline Authors & SG & 1st target & 2nd target & FU monhs & Conclusions & Minors \\
\hline Pérez-Mañanes 2016 [33] & 8 & $62 \%$ & $183-185$ & & Less errors & 18 \\
Munier 2017 [30] & 10 & $182.4^{\circ}$ & & 12 & No control & 12 \\
Kim 2018 [17] & 20 & $57.2-67.5 \%$ & $183^{\circ}-186^{\circ}$ & & Higher accuracy & 16 \\
Yang 2018 [41] & 10 & $62.5 \%$ & & & No control & 10 \\
Chernchujit 2019 [5] & 19 & $185^{\circ}-187^{\circ}$ & & 20 & No control & 12 \\
Donnez 2018 [6] & & $\Delta$ mMPTA & 88 & 24 & No control & 18 \\
Chaouche 2019 [4] & 100 & $\Delta$ HKA & & 12 & No control & 12 \\
Jacquet 2019 [15] & 71 & $\Delta$ HKA & $57.2-67.5 \%$ & & No control & 12 \\
Kuriyama 2019 [21] & 47 & $62.5 \%$ & & No control & 10 \\
Fucentese 2020 [8] & 23 & $62.5 \%$ & Planned HKA 9.7 & & & \\
\hline
\end{tabular}

$S G$ Study group, $F U$ Follow-Up, $H K A$ Hip-Knee Angle, mMPTA mechanical medial proximal tibial angle
Table 2 Coronal correction of the Patient Specific Instrumentation (PSI)/ traditional technique studies the analysis showed that there was no significant difference between the two groups (SMD $=-0.10 ; 95 \% \mathrm{CI}-1.31$ to 1.12; $P=$ n.s.) (Fig. 2). The rate of outliers was $15.9 \%$ in the CAS group and $38.2 \%$ in the traditional group. The pooled results showed that the number of outliers was lower in navigated HTO than that in conventional HTO $(\mathrm{OR}=0.40 ; 95 \%$ CI 0.17 to $0.96 ; P=0.04$ ) (Fig. 3 ).

In the PSI/traditional tecnique studies the rate of outliers was $15 \%$ in the PSI group and $40 \%$ in the traditional group (Table 2). The pooled results showed that there was no significant difference between the two groups $(\mathrm{SMD}=0.01$; $95 \%$ CI -0.58 to $0.59 ; P=0.98$ ) (Fig. 4 ).

\section{Outcomes assessment: duration of surgery and complications}

In the CAS/traditional subgroup the average duration of surgery was much longer in the CAS subgorup (97.4 min), while the rate of complications was lower (7.7\%) (Table 5). The pooled results showed that the risk of complications was lower in navigated HTO than that in conventional HTO $(\mathrm{OR}=0.38 ; 95 \%$ CI 0.10 to $1.45 ; P=0.16)$ (Fig. 5).

In the PSI/traditional subgroup the average duration of surgery was shorter in the PSI subgroup (60.8 min), while the rate of complications was higher $(7.7 \%$ ) (Table 6) 


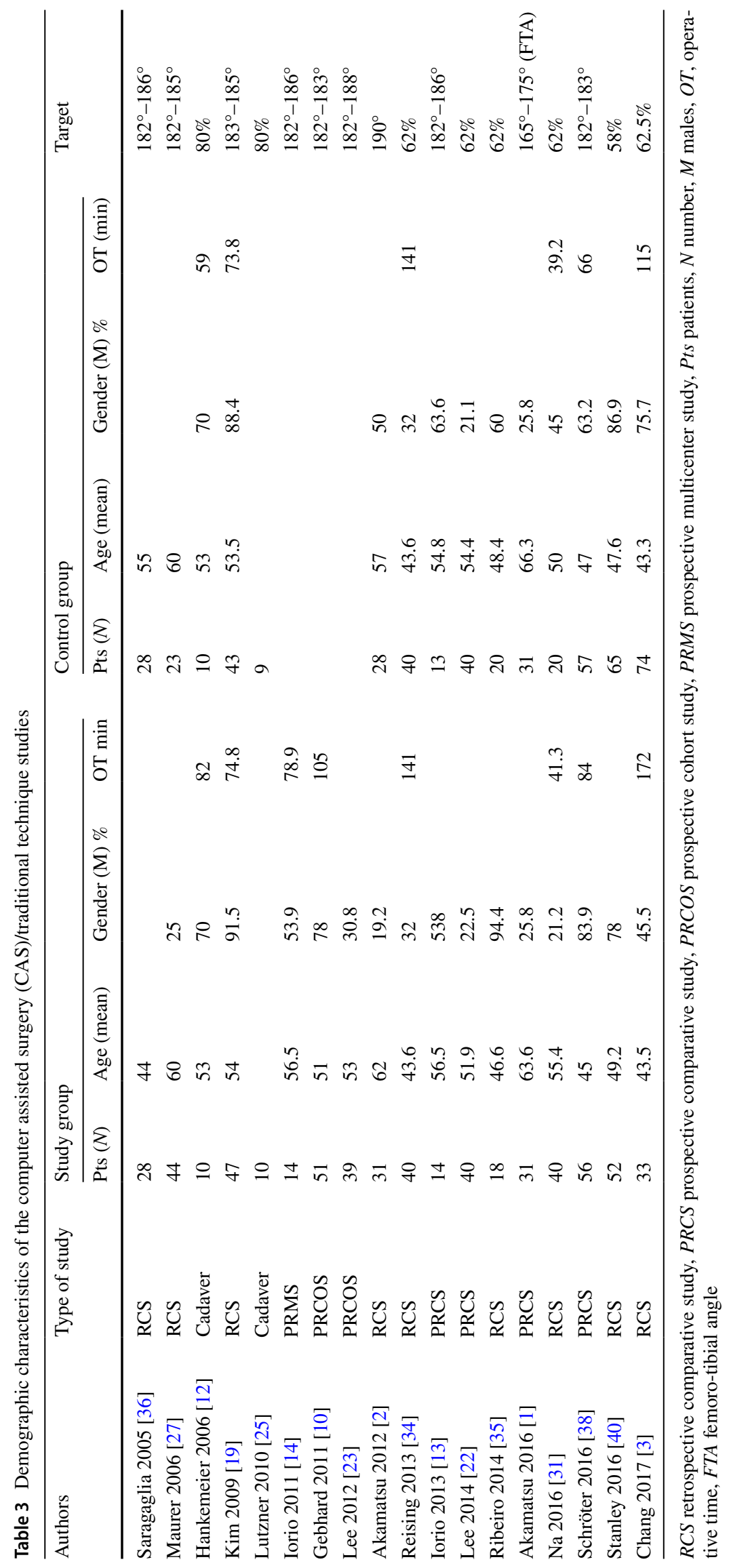


Table 4 Demographic characteristics of the PSI/traditional technique studies

\begin{tabular}{|c|c|c|c|c|c|c|c|c|c|c|}
\hline \multirow[t]{2}{*}{ Authors } & \multirow[t]{2}{*}{ Type of study } & \multicolumn{4}{|c|}{ Study group } & \multicolumn{4}{|c|}{ Control group } & \multirow[t]{2}{*}{ Target } \\
\hline & & $\operatorname{Pts}(N)$ & Age (mean) & $\begin{array}{l}\text { Gender } \\
\mathrm{M}(\%)\end{array}$ & OT (min) & Pts $(N)$ & Age (mean) & $\begin{array}{l}\text { Gender } \\
\mathrm{M}(\%)\end{array}$ & OT (min) & \\
\hline Pérez-Mañanes 2016 [33] & PRCS & 8 & 44 & & 61 & 20 & 45 & & 92 & $62 \%$ \\
\hline Munier 2017 [30] & PRCOS & 10 & 46 & & & & & & & $182.4^{\circ}$ \\
\hline Kim 2018 [17] & RCS & 20 & 55.7 & 4 & & 20 & 55.1 & 3 & & $57.2-67.5 \%$ \\
\hline Yang 2018 [41] & PRCOS & 10 & 67.2 & 4 & & & & & & $62.5 \%$ \\
\hline Chernchujit 2019 [5] & PRCOS & 19 & 46.9 & 11 & 95 & & & & & $185^{\circ}-187^{\circ}$ \\
\hline Donnez 2018 [6] & Cadaver & 88 & 88 & 20 & & & & No & & $\Delta \mathrm{mMPTA}$ \\
\hline Chaouche 2019 [4] & PROCS & 100 & 44.2 & 59 & & & & & & $\Delta \mathrm{HKA}$ \\
\hline Jacquet 2019 [15] & PROCS & 71 & 44.3 & 38 & 26.3 & & & & & $\Delta \mathrm{HKA}$ \\
\hline Kuriyama 2019 [21] & PROCS & 47 & 61.7 & 16 & & & & & & $62.5 \%$ \\
\hline Fucentese 2020 [8] & PRCOS & 23 & 45.2 & 16 & & & & & & $62.5 \%$ \\
\hline
\end{tabular}

$R C S$ retrospective comparative study, PRCS prospective comparative study, $P R C O S$ prospective cohort study, $P t s$ patients, $N$ number, $M$ males, $O T$ operative time, min minutes, $H K A$ hip-knee angle, $m M P T A$ mechanical medial proximal tibial angle

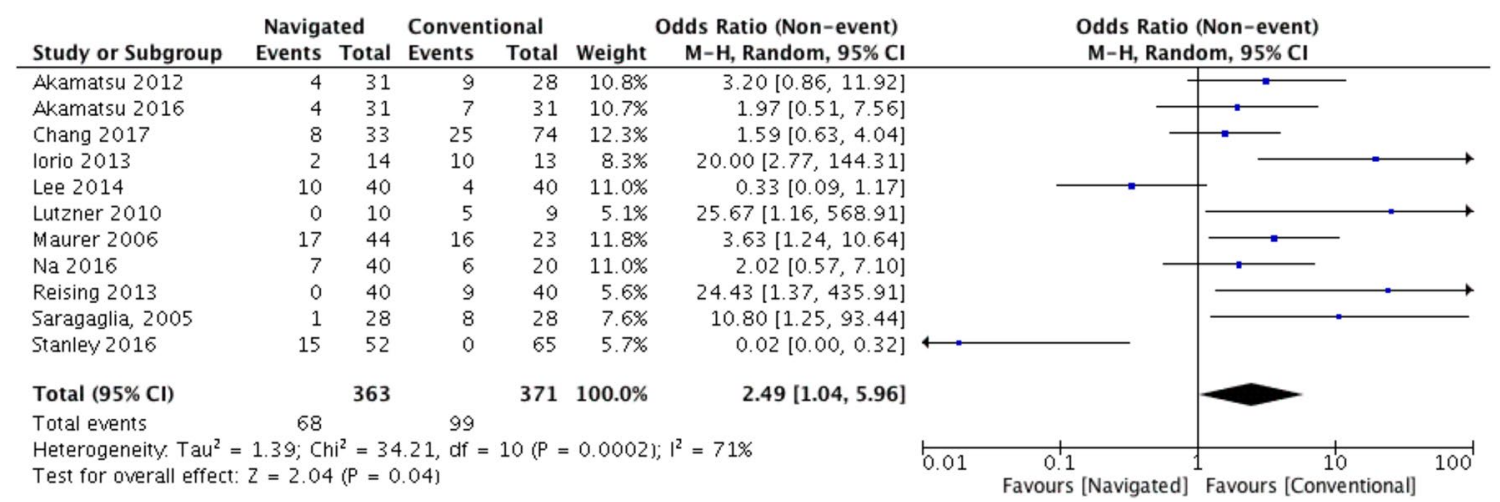

Fig. 3 Comparison of outliers in the computer assisted surgery (CAS)/traditional technique studies

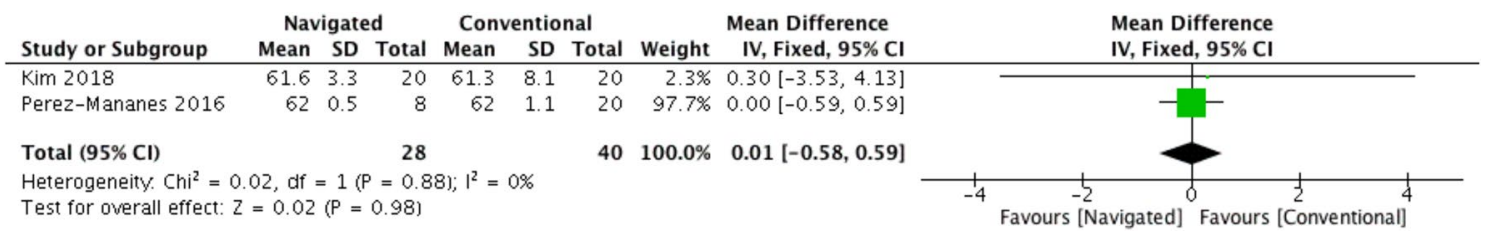

Fig. 4 Comparison of coronal correction in the patient specific instrumentation (PSI)/traditional technique studies

\section{Discussion}

The present study has some notable findings. First of all, the use of CAS and PSI leads to statistically significant reduction of postoperative outliers when compared to traditional technique. Second, they do not lead to a statistically superior accuracy in achieving the target postoperative alignment, although a small trend toward superior results was observed.
Finally there is no conensus on which is the ideal correction target after MOWHTO.

Several studies aimed at angular correction (HKA) with wide angular variability while others aimed at a specific target in the Fujisawa area (Tables 3, 4). Mikulicz-Radecki first proposed in 1880 a straight line (Mikulicz line) connecting the center of the femoral head and the center of the talus. The line gives the overall mechanical alignment of the lower limb. The HKA gives the overall alignment of the lower limb and is the result of three components: the bony alignment 


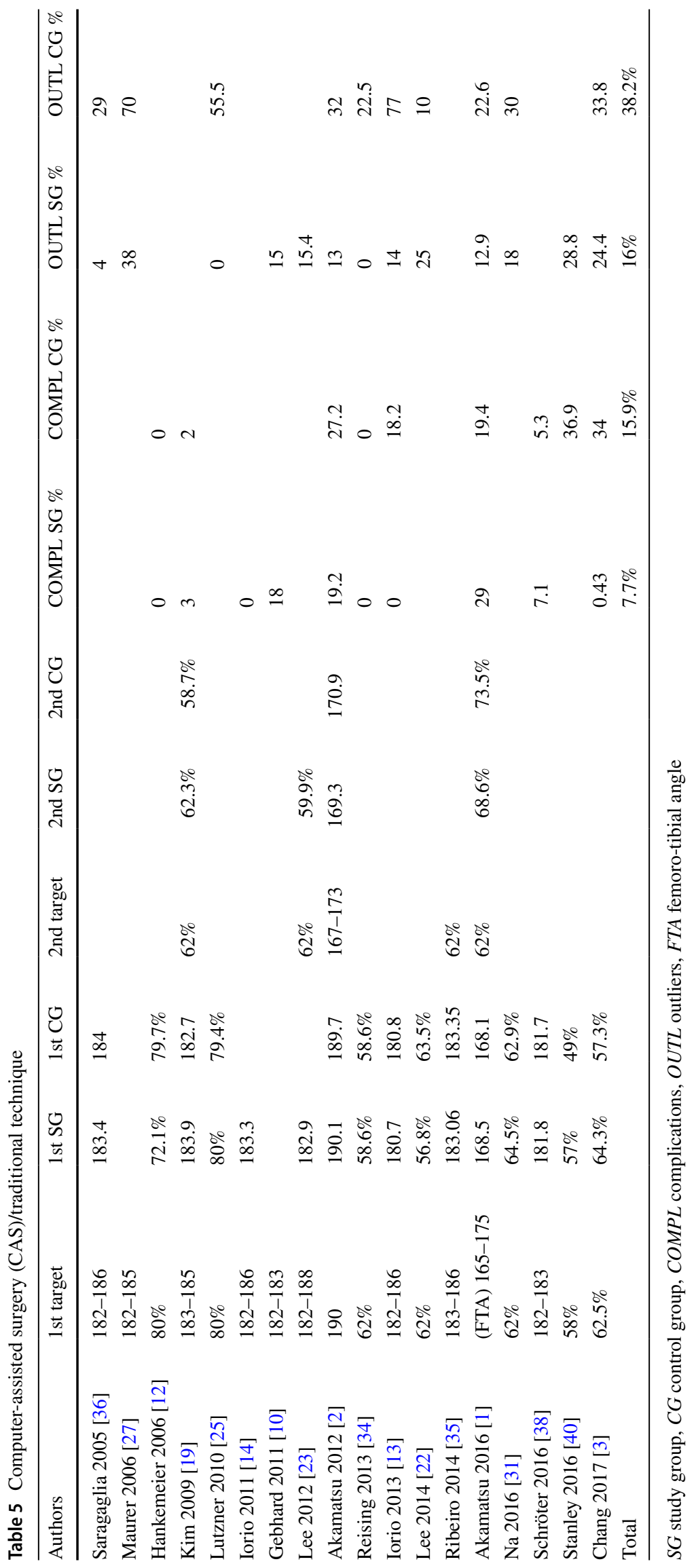




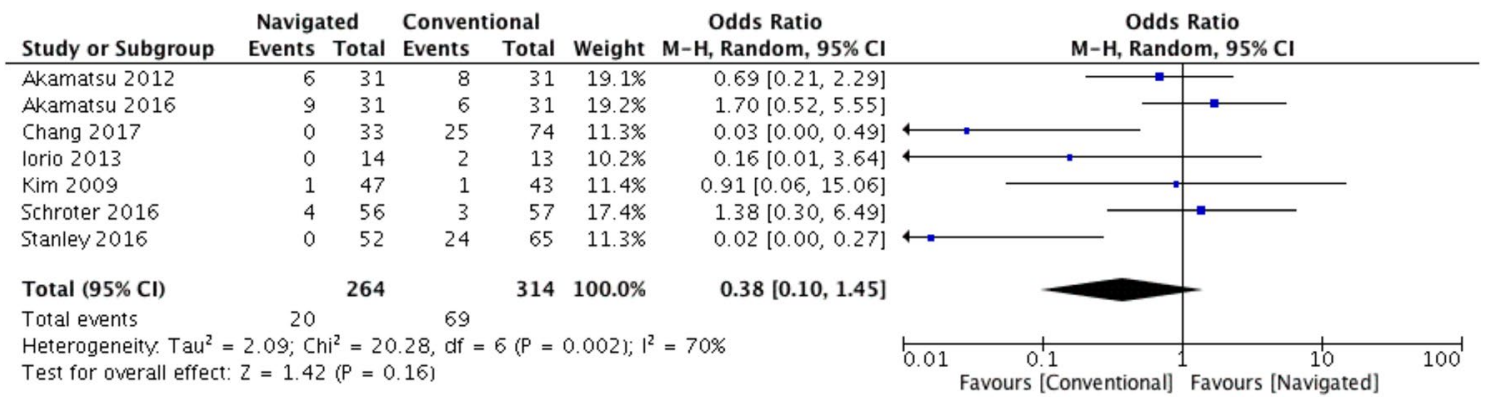

Fig. 5 Comparison of complication rate in the computer assisted surgery (CAS)/traditional technique studies

of the femur and the tibia, the wear of the articular cartilage and the slackness of the capsule/ligaments complex around the knee. However it does not give any information on where the deformity is located (bone, cartilage or ligaments).

The first step of a traditional MOWHTO is to establish the desired postoperative alignment and therefore to plan the desired correction. The planning should be performed on full leg standing weight bearing X-ray [22]. During planning, it is easier to consider the Mikulicz line in relation to the \% of the tibial plateau rather than the HKA. The physiological Mikulicz line crosses the tibial plateau in the geometrical middle, but with a mechanical axis deviation (MAD) of some 4-8 $\mathrm{mm}$ to the medial side [32]. As a results, an intersection at $45 \%$ TPW has to be considered normal while its medial shift is associated with a varus deformity. The most common way to plan the desired correction is to follow the Miniaci and Jakob method [28]. It is based on the necessity to shift the Mikulicz line in a more lateral position to correct the varus deformity. Fujisawa et al. were the first to advocate a postoperative Mikulicz line passing at around $65-70 \%$ of the tibial width [9]. This principle roughly corresponds to a postoperative HKA of $183^{\circ}-185^{\circ}$. Although Dugdale and Noyes hypothesized that different intersection points could provide equivalent outcomes [7], the principles of the correction proposed by Jakob and Miniaci remain accepted. In any case, a shift of $65 \%$ is set as the upper boundary, but for some surgeons, when osteoarthritis is severe, this upper boundary might be extended up to $75 \%$ [26]. On the contrary the general trend is to reduce the shift toward 55-60\% adapting the correction to the individual deformity. This wide variability reflects the different target values found in the present review.

A second aspect emerges from the present review and meta-analysis: the addition of technology do not clearly increases the accuracy of MOWHTO, although a trend toward superior accuracy was observed. The accuracy of bony correction is important; traditional technique has several drawbacks when compared to CAS and PSI. First of all, errors with the traditional technique can result from inappropriate planning. When transferring angular correction into millimeters (of opening or closing wedges) there can be mismatches. This may result from poorly performed $\mathrm{X}$-ray (not real dimensions, malrotations of the limb, poor projections, wrong assessment of the deformity, absence of correction of intraarticular deformity) or poor planning itself (wrong drawings, lines, HKA correction). PSI involves patient specific cutting blocks which are manufactured on patient-specific 3D-CT scan films and therefore this kind of errors are significantly reduced. In addition there can be introperative errors associated with direct visual inspection methods such as the cable method or grid boards [31]. They can also be the consequence of high intra-observer variations and the low reproducibility of intra-operative assessment tools, including fluoroscopy-based methods [23, 26]. Both the CAS and PSI are advantageous in reducing the rate and amount of these errors since they guarantee objective intraoperative references and data. Several previous studies have shown that navigation assisted HTO is superior in terms of reducing outliers from the predicted postoperative limb alignment [10, 11, 23, 34]. In a similar way the use of patient specific instrumentation (PSI) can be a reliable option to improve the accuracy of HTOs [15]. In the present review several studies compared the accuracy of coronal alignment between the traditional technique and the CAS. Although a trend toward increased accuracy was observed in the CAS group, the pooled results including subgroup of angular correction (mean \pm SD) and area (\%) described by Fujisawa showed that there was no statistically significant difference between the two groups. On the contrary a significant reduction of outliers was observed when CAS was used, although some authors still reported no differences in their series [22, $34,35,38,40]$. Less studies analyzed the accuracy of PSI and only 2 compared this technique to traditional surgery $[17,33]$. The pooled results including subgroup of angular correction (mean \pm SD) and area (\%) described by Fujisawa showed that there was no statistically significant difference between the two groups.

The present study has some notable limitations. Firstly, although several databases have been accessed with different combination of appropriate keywords, it is possible that 


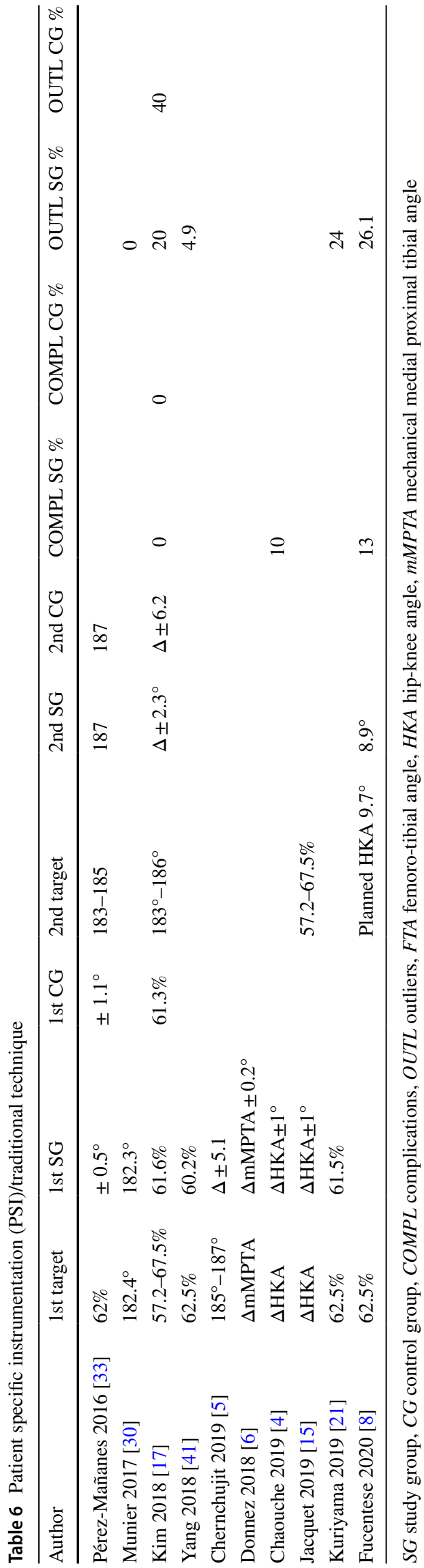

some articles may not have been included in our search. Second, the level of evidence of the included studies was not high because the majority were observational comparative studies rather than randomized controlled. Third, almost all of the available articles in the PSI subgroup are case series with no control group; this could reduce the scientific relevance of the reported data. Fourtly, the analysis of postoperative alignment should be performed at the same time in the different studies.

Although the reported limitations the present study yields some relevant informations for daily practice First of all, CAS and PSI ensure a statistically significant reduction in the rate of postoperative outliers. Second, they do not lead to a statistically superior accuracy in achieving the target postoperative alignment, although a small trend toward superior results was observed. Finally, a lack of consensus on which is the ideal postoperative alignement target clearly emerges from the present review.

\section{Conclusions}

New technologies such as CAS and PSI yield reduced rate of outliers after MOWHTO when compared to the traditional surgical technique. Coversely they do not lead to statistically significant improvement in the accuracy of postoperative alignment, At the same time, it is clear that no consensus exists on the ideal correction target to achieve after surgery, in terms of angular corrections (HKA) or target zone on the tibial plateau (Fujisawa area and Mikulicz line).

\section{Compliance with ethical standards}

Conflict of interest The authors declare that they have no conflicts of interest.

Funding No funding has been received for this study.

Ethical approval Ethical approval was not required for this study.

\section{References}

1. Akamatsu Y, Kobayashi H, Kusayama Y, Kumagai K, Saito T (2016) Comparative study of opening-wedge high tibial osteotomy with and without a combined computed tomography-based and image-free navigation system. Arthroscopy 32(10):2072-2081

2. Akamatsu Y, Mitsugi N, Mochida Y, Taki N, Kobayashi H, Takeuchi R, Saito T (2012) Navigated opening wedge high tibial osteotomy improves intraoperative correction angle compared with conventional method. Knee Surg Sports Traumatol Arthrosc 20(3):586-593 
3. Chang J, Scallon G, Beckert M, Zavala J, Bollier M, Wolf B, Albright J (2017) Comparing the accuracy of high tibial osteotomies between computer navigation and conventional methods. Comput Assist Surg 22(1):1-8

4. Chaouche S, Jacquet C, Fabre-Aubrespy M, Sharma A, Argenson JN, Parratte S, Ollivier M (2019) Patient-specific cutting guides for open-wedge high tibial osteotomy: safety and accuracy analysis of a hundred patients continuous cohort. Int Orthop 43(12):2757-2765

5. Chernchujit B, Tharakulphan S, Prasetia R, Chantarapanich N, Jirawison C, Sitthiseripratip K (2019) Preoperative planning of medial opening wedge high tibial osteotomy using 3D computeraided design weight-bearing simulated guidance: technique and preliminary result. J Orthop Surg 27(1):2309499019831455

6. Donnez M, Ollivier M, Munier M, Berton P, Podgorski JP, Chabrand P, Parratte S (2018) Are three-dimensional patientspecific cutting guides for open wedge high tibial osteotomy accurate? An in vitro study. J Orthop Surg Res 13(1):171

7. Dugdale TW, Noyes FR, Styer D (1992) Preoperative planning for high tibial osteotomy. The effect of lateral tibiofemoral separation and tibiofemoral length. Clin Orthop Relat Res 274:248-264

8. Fucentese SF, Meier P, Jud L, Köchli GL, Aichmair A, Vlachopoulos L, Fürnstahl P (2020) Accuracy of 3D-planned patient specific instrumentation in high tibial open wedge valgisation osteotomy. J Exp Orthop 7(1):7

9. Fujisawa Y, Masuhara K, Shiomi S (1979) The effect of high tibial osteotomy on osteoarthritis of the knee An arthroscopic study of 54 knee joints. Orthop Clin N Am 10(3):585-608

10. Gebhard F, Krettek C, Hufner T, Grutzner PA, Stockle U, Imhoff AB, Lorenz S, Ljungqvist J, Keppler P (2011) Reliability of computer-assisted surgery as an intraoperative ruler in navigated high tibial osteotomy. Arch Orthop Trauma Surg 131(3):297-302

11. Han SB, Kim HJ, Lee DH (2017) Effect of computer navigation on accuracy and reliability of limb alignment correction following open-wedge high tibial osteotomy: a meta-analysis. Biomed Res Int 2017:3803457

12. Hankemeier S, Hufner T, Wang G, Kendoff D, Zeichen J, Zheng G, Krettek C (2006) Navigated open-wedge high tibial osteotomy: advantages and disadvantages compared to the conventional technique in a cadaver study. Knee Surg Sports Traumatol Arthrosc 14(10):917-921

13. Iorio R, Pagnotelli M, Vadalà A, Giannetti S, Di Sette P, Papandrea P, Conteduca F, Ferretti A (2013) Open-wedge high tibial osteotomy:comparison between manual and computer-assisted techniques. Knee Surg Sports Traumatol Arthrosc 21(1):113-119

14. Iorio R, Vadalà A, Giannetti S, Pagnotelli M, Di Sette P, Conteduca F, Ferretti A (2010) Computer-assisted high tibial osteotomy: preliminary results. Orthopedics 33(10):82-86

15. Jacquet C, Sharma A, Fabre M, Ehlinger M, Argenson JN, Parratte S, Ollivier M (2019) Patient-specific high-tibial osteotomy's cutting-guides decrease operating time and the number of fluoroscopic images taken after a brief learning curve. Knee Surg Sports Traumatol Arthrosc. https://doi.org/10.1007/s00167-019-05637-6

16. Jung WH, Takeuchi R, Chun CW, Lee JS, Jeong JH (2015) Comparison of results of medial opening-wedge high tibial osteotomy with and without subchondral drilling. Arthroscopy 31(4):673-679

17. Kim HJ, Park J, Shin JY, Park IH, Park KH, Kyung HS (2018) More accurate correction can be obtained using a three-dimensional printed model in open-wedge high tibial osteotomy. Knee Surg Sports Traumatol Arthrosc 26(11):3452-3458

18. Kim KI, Seo MC, Song SJ, Bae DK, Kim DH, Lee SH (2017) Change of chondral lesions and predictive factors after medial open-wedge high tibial osteotomy with a locked plate system. Am J Sports Med 45(7):1615-1621
19. Kim SJ, Koh YG, Chun YM, Kim YC, Park YS, Sung CH (2009) Medial opening wedge high-tibial osteotomy using a kinematic navigation system versus a conventional method: a 1 year retrospective, comparative study. Knee Surg Sports Traumatol Arthrosc 17(2):128-134

20. Koshino T, Morii T, Wada J, Saito H, Ozawa N, Noyori K (1989) High tibial osteotomy with fixation by a blade plate for medial compartment osteoarthritis of the knee. Orthop Clin N Am 20(2):227-243

21. Kuriyama S, Morimoto N, Shimoto T, Takemoto M, Nakamura S, Nishitani K, Ito H, Matsuda S, Higaki H (2019) Clinical efficacy of preoperative 3D planning for reducing surgical errors during open-wedge high tibial osteotomy. J Orthop Res 37(4):898-907

22. Lee DH, Han SB, Oh KJ, Lee JS, Kwon JH, Kim JI, Patnaik S, Shetty GM, Nha KW (2014) The weight-bearing scanogram technique provides better coronal limb alignment than the navigation technique in open high tibial osteotomy. Knee 21(2):451-455

23. Lee DH, Nha KW, Park SJ, Han SB (2012) Preoperative and postoperative comparisons of navigation and radiologic limb alignment measurements after high tibial osteotomy. Arthroscopy 28:1842-1850

24. Li Y, Zhang H, Zhang J, Li X, Song G, Feng H (2015) Clinical outcome of simultaneous high tibial osteotomy and anterior cruciate ligament reconstruction for medial compartment osteoarthritis in young patients with anterior cruciate ligament-deficient knees: a systematic review. Arthroscopy 31(3):507-519

25. Lutzner J, Gross AF, Gunther KP, Kirschner S (2010) Precision of navigated and conventional open-wedge high tibial osteotomy in a cadaver study. Eur J Med Res 30 15(3):117-120

26. Marti CB, Gautier E, Watchtl SW, Jakob RP (2004) Accuracy of frontal and sagittal plane correction in open-wedge high tibial osteotomy. Arthroscopy 20:366-372

27. Maurer F, Wassmer G (2006) High tibial osteotomy: does navigation improve results? Orthopedics 29(10):S130-S132

28. Miniaci A, Ballmer FT, Ballmer PM, Jakob RP (1989) Proximal tibial osteotomy. A new fixation device. Clin Orthop Relat Res 246:250-259

29. Moher D, Liberati A, Tetzlaff Altman DG (2014) Preferred reporting items for systematic reviews and meta-analyses. Ann Intern Med 151:264-269

30. Munier M, Donnez M, Ollivier M, Flecher X, Chabrand P, Argenson JN, Parratte S (2017) Can three-dimensional patientspecific cutting guides be used to achieve optimal correction for high tibial osteotomy? Pilot study. Orthop Traumatol Surg Res 103(2):245-250

31. Na YG, Eom SH, Kim SJ, Chang MJ, Kim TK (2016) The use of navigation in medial opening wedge high tibial osteotomy can improve tibial slope maintenance and reduce radiation exposure. Int Orthop 40(3):499-507

32. Paley D, Tetsworth K (1992) Mechanical axis deviation of the lower limbs. Preoperative planning of uniapical angular deformities of the tibia or femur. Clin Orthop Relat Res 280:48-64

33. Pérez-Mañanes R, Burrò JA, Manaute JR, Rodriguez FC, Martin JV (2016) 3D Surgical printing cutting guides for open-wedge high tibial osteotomy: do it yourself. J Knee Surg 29(8):690-695

34. Reising K, Strohm PC, Hauschild O, Schmal H, Khattab M, Sudkamp NP, Niemeyer P (2013) Computer-assisted navigation for the intraoperative assessment of lower limb alignment in high tibial osteotomy can avoid outliers compared with the conventional technique. Knee Surg Sports Traumatol Arthrosc 21(1):181-188

35. Ribeiro Ch, Severino NR, de Barros Moraes, Fucs PM (2014) Opening wedge high tibial osteotomy: navigation system compared to the conventional technique in a controlled clinical study. Int Orthop 38(8):1627-1631 
36. Saragaglia D, Roberts J (2005) Navigated osteotomies around the knee in 170 patients with osteoarthritis secondary to genu varum. Orthopedics 28(10):s1269-s1274

37. Schallberger A, Jacobi M, Wahl P, Maestretti G, Jakob RP (2011) High tibial valgus osteotomy in unicompartmental medial osteoarthritis of the knee: a retrospective follow-up study over 13-21 years. Knee Surg Sports Traumatol Arthrosc 19(1):122-127

38. Schröter S, Ihle C, Elson DW, Döbele S, Stöckle U, Ateschrang A (2016) Surgical accuracy in high tibial osteotomy: coronal equivalence of computer navigation and gap measurement. Knee Surg Sports Traumatol Arthrosc 24(11):3410-3417

39. Slim K, Nini E, Forestier D, Kwiatkowski F, Panis Y, Chipponi J (2003) Methodological index for non-randomized studies (MINORS): development and validation of a new instrument. ANZ J Surg 73(9):712-716
40. Stanley JC, Robinson KG, Devitt BM, Richmond AK, Webster KE, Whitehead TS, Feller JA (2016) Computer assisted alignment of opening wedge high tibial osteotomy provides limited improvement of radiographic outcomes compared to fluoroscopic alignment. Knee 23(2):289-294

41. Yang JC, Chen CF, Luo CA, Chang MC, Lee OK, Huang Y, Lin SC (2018) Clinical experience using a 3D-printed patient-specific instrument for medial opening wedge high tibial osteotomy. Biomed Res Int. https://doi.org/10.1155/2018/9246529 